\title{
Finite versus zero-temperature hysteretic behavior of spin glasses: Experiment and theory
}

\author{
Helmut G. Katzgraber, ${ }^{1}$ Didier Hérisson, ${ }^{2}$ Michael Östh, ${ }^{2}$ Per Nordblad, ${ }^{2}$ Atsuko Ito, ${ }^{3}$ and Hiroko Aruga Katori ${ }^{3}$ \\ ${ }^{1}$ Theoretische Physik, ETH Zürich, CH-8093 Zürich, Switzerland \\ ${ }^{2}$ Department of Engineering Sciences, Uppsala University, Box 534, SE-751 21 Uppsala, Sweden \\ ${ }^{3}$ Riken, Hirosawa 2-1, Wako, Saitama, 351-0198, Japan
}

(Dated: November 13, 2018)

\begin{abstract}
We present experimental results attempting to fingerprint nonanalyticities in the magnetization curves of spin glasses found by Katzgraber et al. [Phys. Rev. Lett. 89, 257202 (2002)] via zerotemperature Monte Carlo simulations of the Edwards-Anderson Ising spin glass. Our results show that the singularities at zero temperature due to the reversal-field memory effect are washed out by the finite temperatures of the experiments. The data are analyzed via the first order reversal curve (FORC) magnetic fingerprinting method. The experimental results are supported by Monte Carlo simulations of the Edwards-Anderson Ising spin glass at finite temperatures which agree qualitatively very well with the experimental results. This suggests that the hysteretic behavior of real Ising spinglass materials is well described by the Edwards-Anderson Ising spin glass. Furthermore, reversalfield memory is a purely zero-temperature effect.
\end{abstract}

PACS numbers: $75.50 . \mathrm{Lk}, 75.40 . \mathrm{Mg}, 05.50 .+\mathrm{q}$

\section{INTRODUCTION}

While the nonequilibrium behavior of spin glasses $1,2,3$ has been studied in detail and several aspects have been applied to fields as wide as biology and financial analysis, less work $4,5,6,7,8$ has been done to understand the hysteretic behavior ${ }^{-9}$ of spin glasses in a time-dependent field. Because the understanding of hysteretic systems plays a prominent role in the development of magnetic recording media, understanding fundamental properties of simple hysteretic systems such as spin glasses is of paramount importance.

In Ref. 8 Katzgraber et al. reported on a novel memory effect - the reversal-field memory effect-found in spinglass systems: When the field is decreased from saturation to a reversal field $H_{\mathrm{R}}$, upon return to saturation the magnetization exhibits a singularity (kink) at $H=-H_{\mathrm{R}}$. The underlying spin-reversal symmetry of the Hamiltonian is the source of this effect which was observed first numerically at zero temperature for the two-dimensional

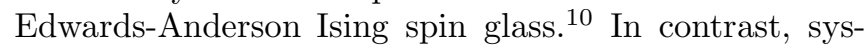
tems which do not possess spin-reversal symmetry, such as the random-field Ising model,,$\frac{5,6}{6}$ do not exhibit this memory effect. Because the kinks in the magnetization curves are easily overlooked, Katzgraber et al. used the first order reversal curve (FORC) method ${ }^{8,11,12}$ to characterize the reversal-field memory effect. In a FORC diagram, the kinks in the magnetization curves are captured by a pronounced vertical ridge along the coercivity axis (see below).

Using the superb fingerprinting abilities of the FORC method we have attempted to experimentally detect the reversal-field memory effect in $\mathrm{Fe}_{0.5} \mathrm{Mn}_{0.5} \mathrm{TiO}_{3}$ Ising spin-glass samples. Our finite-temperature experimental results show that the reversal-field memory effect is not present. Moreover, these results are verified via finite-temperature nonequilibrium Monte Carlo simula- tions of the three-dimensional Edwards-Anderson Ising spin glass. The numerical results show that the reversalfield memory effect can only be observed at experimentally inaccessible temperatures. The good agreement between the simulation and experiments suggest that the hysteretic properties of $\mathrm{Fe}_{0.5} \mathrm{Mn}_{0.5} \mathrm{TiO}_{3}$ can be well modeled using the short-range Edwards-Anderson Ising spin glass model.

The paper is structured as follows. First we review the FORC method in Sec. III In Sec. III we present experimental results on $\mathrm{Fe}_{0.5} \mathrm{Mn}_{0.5} \mathrm{TiO}_{3}$. In Sec. IV results of zero- and finite-temperature Monte Carlo simulations of the three-dimensional Edwards-Anderson Ising spin glass are presented, followed by concluding remarks in Sec. V.

\section{OUTLINE OF THE FORC METHOD}

FORC diagrams, $, 11,12$ which can be viewed as a model-independent generalization of Preisach diagrams ${ }^{13,14}$ complement current methods to characterize magnetic interactions in hysteretic systems, $, 15,16,17$ such as the $\delta M$ method $\underline{18,19}$ The advantage of the FORC method over other approaches is the extreme sensitivity to microscopic details of hysteretic systems. Although the wealth of information a FORC diagram delivers still remains to be fully characterized, the method can be used as an extremely sensitive "fingerprint" of a hysteretic system.

To calculate a FORC diagram, a family of FORCs with different reversal fields $H_{\mathrm{R}}$ is measured either experimentally or numerically $\underline{20}$ The measurements start at saturation and the field is then reversed at different values of the reversal field $H_{\mathrm{R}}$. The mixed second-order derivative 11.12 of the magnetization $M\left(H, H_{\mathrm{R}}\right)$ as a function of the applied and reversal field yields the FORC 


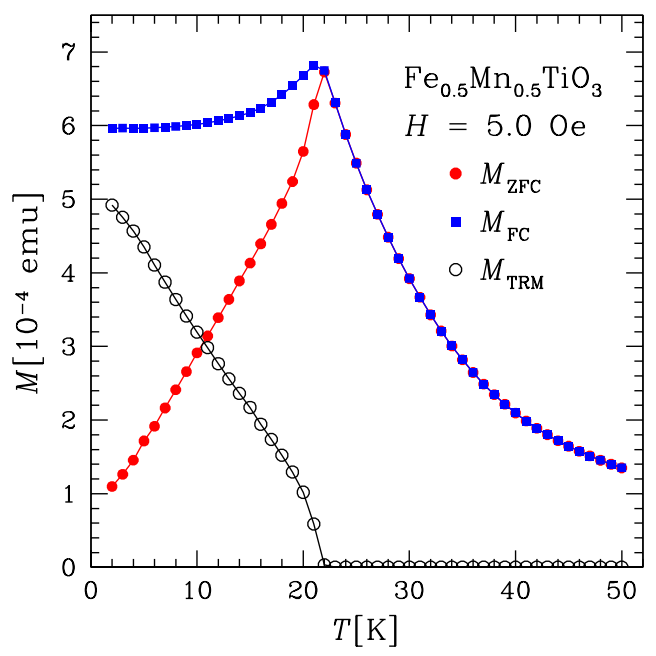

FIG. 1: (Color online) Zero field cooled (ZFC), field cooled (FC) and thermoremanent magnetization (TRM) of the $\mathrm{Fe}_{0.5} \mathrm{Mn}_{0.5} \mathrm{TiO}_{3}$ sample as a function of temperature $T$ for an applied field of $H=5 \mathrm{Oe}$. For this experimental sample the glass transition occurs at $T_{\mathrm{c}} \approx 21 \mathrm{~K}$.

diagram $\rho\left(H, H_{\mathrm{R}}\right)$ given by

$$
\rho\left(H, H_{\mathrm{R}}\right)=-\frac{1}{2}\left[\partial^{2} M / \partial H \partial H_{\mathrm{R}}\right] .
$$

In general, a rotation of the coordinates to

$$
\begin{aligned}
& H_{\mathrm{c}}=\left[H-H_{\mathrm{R}}\right] / 2, \\
& H_{\mathrm{b}}=\left[H+H_{\mathrm{R}}\right] / 2,
\end{aligned}
$$

the local coercivity $H_{\mathrm{c}}$ and bias $H_{\mathrm{b}}$, respectively, yields the FORC distribution $\rho\left(H_{\mathrm{b}}, H_{\mathrm{c}}\right)$. Therefore, under the assumption that a hysteretic system can be described by microscopic switching units, each of which have a given coercivity $H_{\mathrm{c}}$ and a bias field $H_{\mathrm{b}}$, the FORC diagram corresponds to the distribution of these microscopic bias/coercivity fields. In the case of discrete spin models such as the random-field Ising model,,$\frac{5,6}{, 6}$ these microscopic switching units can, in general, be identified with the simulated spins $\stackrel{21}{=}$ This is not the case for systems which exhibit frustration, such as the Edwards-Anderson Ising spin-glass model. FORC diagrams have been applied to a variety of hysteretic systems and fields ranging from geological applications 12 to magnetic materials,,$\frac{822,23}{2}$ as well as more exotic systems

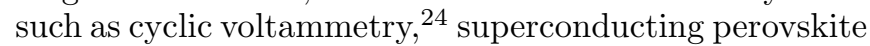
materials, $\stackrel{25}{r}$ and thermal hysteresis ${ }^{26}$

\section{EXPERIMENTAL RESULTS}

The experiments have been performed on a single crystal of the Ising spin glass $\mathrm{Fe}_{0.5} \mathrm{Mn}_{0.5} \mathrm{TiO}_{3}$ in a

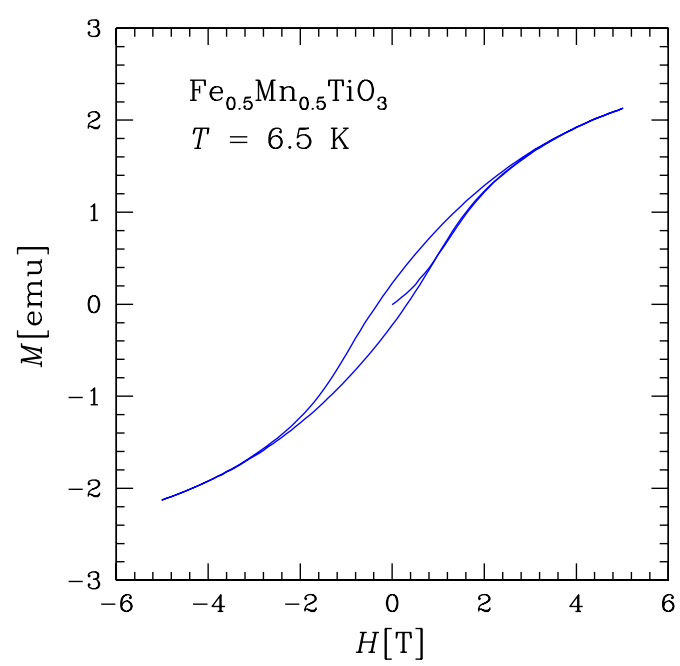

FIG. 2: (Color online) Hysteresis curve of $\mathrm{Fe}_{0.5} \mathrm{Mn}_{0.5} \mathrm{TiO}_{3}$ at $6.5 \mathrm{~K}$, which corresponds to a temperature of $T \approx 0.31 T_{\mathrm{c}}$ (see Fig. 1).

Quantum Design MPMS-XL 5T SQUID (superconducting quantum interference device) magnetometer. Lowfield magnetization vs temperature curves in zero field cooled (ZFC), field cooled (FC), and thermoremanent magnetization (TRM) protocols for $\mathrm{Fe}_{0.5} \mathrm{Mn}_{0.5} \mathrm{TiO}_{3}$ are shown in Fig. 1. The spin-glass transition temperature of $\mathrm{Fe}_{0.5} \mathrm{Mn}_{0.5} \mathrm{TiO}_{3}$ is $T_{\mathrm{c}} \approx 21 \mathrm{~K}$. These measurements are made with the applied field parallel to the $c$ axis of the sample - the corresponding curves measured perpendicular to the field show only a weakly temperature-dependent paramagnetic response, no irreversibility between the ZFC and FC magnetization and zero remanence 27 In addition, in Fig. 2 a magnetization $(M)$ vs applied field $(H)$ hysteresis curve measured at 6.5 $\mathrm{K}$, the temperature of the FORC experiment described below, is shown. An extensive study of the hysteretic behavior of $\mathrm{Fe}_{0.5} \mathrm{Mn}_{0.5} \mathrm{TiO}_{3}$ has been reported by Ito et al. in Ref. 28 .

The FORC measurements have been made at as low a temperature as possible to minimize influences on the FORC distribution from thermal relaxation effects. The temperature must, however, be high enough for the sample to have a reversible magnetization at the highest magnetic field available $(5 \mathrm{~T})$. The chosen working temperature of $6.5 \mathrm{~K}\left(T / T_{\mathrm{c}} \approx 0.31\right)$ optimally fulfills these two requirements.

The derived FORC distribution is shown in Fig. 3, The individual FORCs behind the distribution have been measured using field steps of $80 \mathrm{mT}$ and a limiting field of $\pm 5 \mathrm{~T}$. The measurements are made at a constant field increase rate governed by the time it takes to change the field and record one data point (each data point must be recorded at constant field in the SQUID). To avoid some of the influence of relaxation of the magnetization after decreasing the field from the initial high value to 


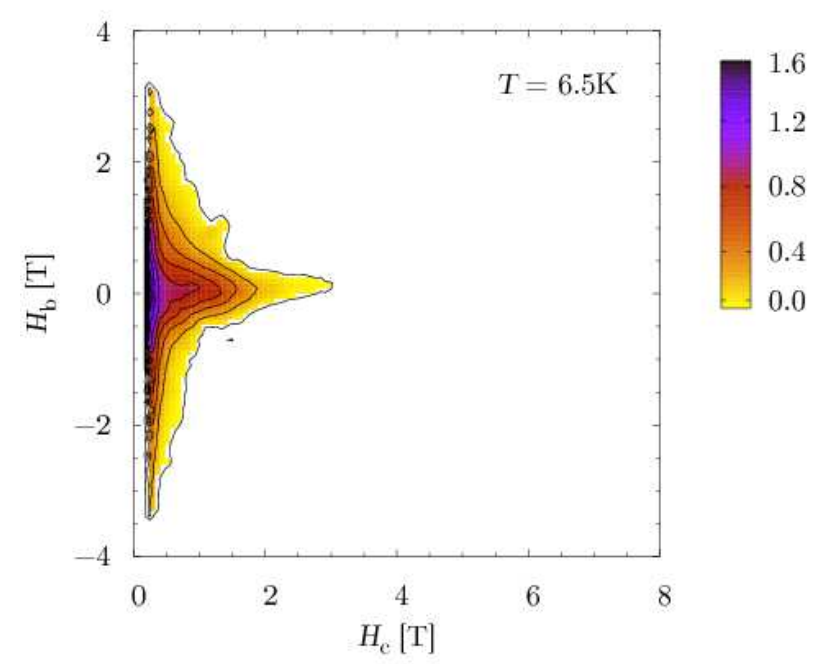

FIG. 3: (Color online) FORC diagram of $\mathrm{Fe}_{0.5} \mathrm{Mn}_{0.5} \mathrm{TiO}_{3}$ at $T=6.5 \mathrm{~K}\left(T / T_{\mathrm{c}} \approx 0.31\right)$. Note that the distribution is not normalized.

$H_{\mathrm{R}}$, the sample is kept for $1000 \mathrm{~s}$ at the reversal field before the actual recording of data during field increase starts. A preliminary report on these results is found in Ref. 29. It can be noted from Fig. 3 that there is a wide distribution of both coercivity and bias fields. The distribution shows a smooth variation with a weak maximum at a coercivity of about $1 \mathrm{~T}$ and a bias field close to zero; and it only slowly decays to zero from a broad ridge along the coercivity axis. In particular, the horizontal ridge reminiscent of the reversal-field memory effect ${ }^{8}$ along the $H_{\mathrm{c}}$ axis has "melted" in comparison to the zerotemperature results from Monte Carlo simulations, and the distribution has broadened along the vertical $H_{\mathrm{b}}$ axis. By comparing to Monte Carlo simulations at finite temperatures (see below) we therefore conclude that finite temperatures and therefore fluctuations of the magnetic moments disrupt the reversal-field memory effect considerably even at temperatures considerably lower than the transition temperature of the glass phase.

\section{NUMERICAL RESULTS}

The experimental results presented in Sec. III do not agree with the numerical results presented in Ref. 8. In particular, the narrow ridge along the horizontal axis that captures the reversal-field memory of the EdwardsAnderson Ising spin glass is washed out (see Fig. 4). To better understand these findings, we simulate the threedimensional Edwards-Anderson Ising spin glass ${ }^{1,10}$ given by the Hamiltonian

$$
\mathcal{H}=\sum_{\langle i, j\rangle} J_{i j} S_{i} S_{j}-H \sum_{i} S_{i}
$$

at finite temperature. Here $S_{i} \in\{ \pm 1\}$ represent Ising spins on a cubic lattice of size $N=L^{3}$ with periodic

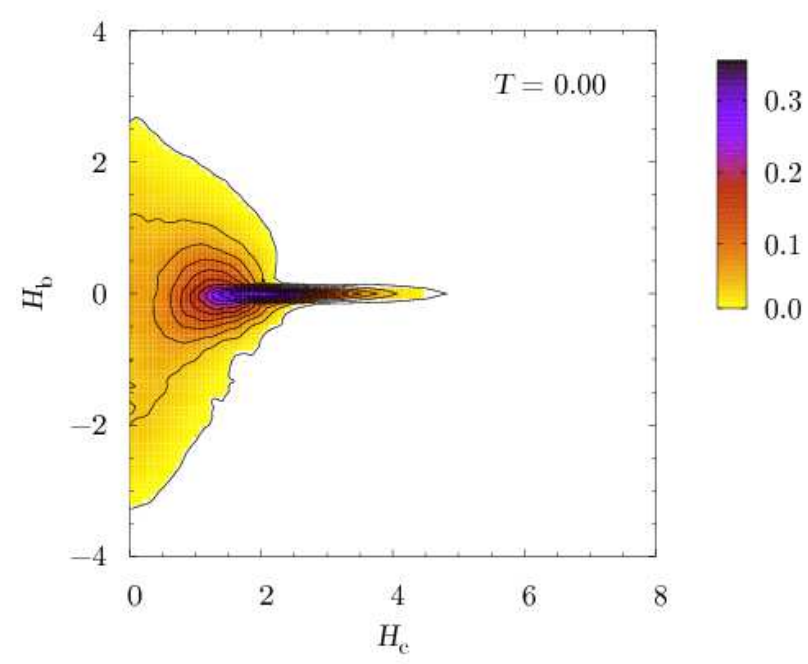

FIG. 4: (Color online) FORC diagram of the threedimensional Edwards-Anderson Ising spin glass at zero temperature (see Ref. 8 for further details). The data show a pronounced ridge at zero bias $\left(H_{\mathrm{b}}=0\right)$ due to the reversal-field memory effect which is a manifestation of the spin-reversal symmetry of the underlying Hamiltonian.

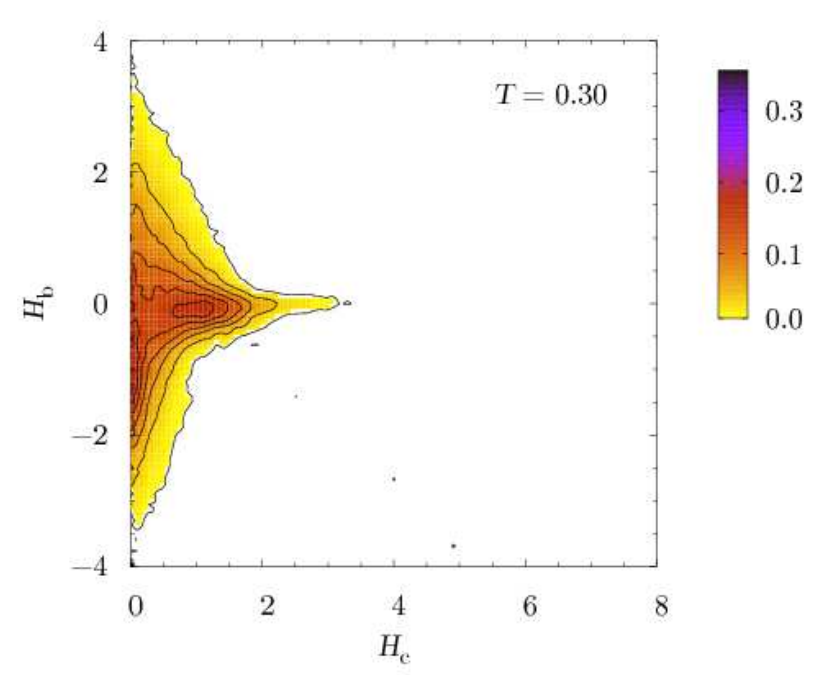

FIG. 5: (Color online) FORC diagram of the threedimensional Edwards-Anderson Ising spin glass at $T=0.30$, which corresponds to $T / T_{\mathrm{c}} \approx 0.31$. The sharp ridge found at zero temperature (Fig. 4) is washed out completely yet the asymmetry along the horizontal axis remains, in very good agreement with the experimental data shown in Fig. 3

boundary conditions. The nearest-neighbor interactions between the spins $J_{i j}$ are chosen from a Gaussian disorder distribution with zero mean and standard deviation unity. $H$ represents an externally applied field. The model has a spin-glass transition at $T_{\mathrm{c}} \approx 0.95 . \underline{30}$

The zero-temperature dynamics of the EdwardsAnderson Ising spin glass is simulated by changing the external field $H$ in small steps starting from positive sat- 
uration. After each field step we compute the local field $h_{i}$ of each spin

$$
h_{i}=\sum_{j} J_{i j} S_{j}-H .
$$

A spin is unstable if it points opposite to its local field, i.e., if $h_{i} S_{i}<0$. Randomly chosen unstable spins are flipped and their local fields updated until all spins are stable. ${ }^{31}$

At finite temperatures the system is simulated with a

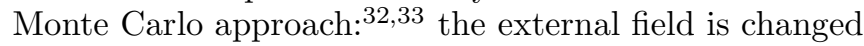
in small steps and for each field step the system is equilibrated using heat-bath Monte Carlo. Tests show that for 1000 Monte Carlo lattice sweeps at $T=0.30$ we obtain hysteresis loops which are independent of Monte Carlo time within error bars. While the system is not in full equilibrium ${ }^{34}$ neither are the experiments and so we argue that our results describe the intrinsic nonequilibrium nature of the finite-temperature experiments correctly. For both zero and finite temperatures we choose a saturation field $H_{\text {sat }}=16.0$ and perform 400 field steps. The presented data for $20^{3}$ spins are averaged over 2000 disorder realizations.

Figure 4 shows a numerical FORC diagram at zero temperature for the Edwards-Anderson Ising spin glass (see Ref.8). The data show a pronounced ridge at $H_{\mathrm{b}}=0$ which is reminiscent of the reversal-field memory effect. In Fig. 5 we present data at $T=0.30$, a temperature (in dimensionless units) which agrees with the values used in the experiments presented in Sec. III The sharp horizontal ridge is completely washed out in very good qualitative agreement with the experimental results and shows that for the Edwards-Anderson Ising spin glass reversal-field memory is destroyed by finite-temperature fluctuations. This suggests that the reversal-field memory effect 8 can only be probed at temperatures much lower than can currently be achieved experimentally.

\section{CONCLUSIONS}

The hysteresis behavior of spin glasses is governed by temperature-relaxation phenomena and field sweep rates dictate the width and shape of the hysteresis loop at finite temperatures. We find fundamental differences between the field-driven hysteresis derived at zero temperature by Monte Carlo simulations and the experimentally as well as numerically observed behavior at finite temperature. In particular, the FORC distributions of finite-temperature Monte Carlo simulations and experiments on $\mathrm{Fe}_{0.5} \mathrm{Mn}_{0.5} \mathrm{TiO}_{3}$ (an Ising spin glass material) at $T / T_{\mathrm{c}} \approx 0.31$ agree qualitatively well (see Figs. 5 ] and 3). The zero-temperature behavior which is dominated by the reversal-field memory effect and characterized by a sharp ridge along the horizontal axis of the FORC diagram is absent at finite temperatures.

Our results therefore show that the reversal-field memory effect can only be observed at temperatures close to or equal to zero which are inaccessible experimentally with current technology. Furthermore, the qualitative agreement between the finite-temperature Monte Carlo data and the experimental results suggest that $\mathrm{Fe}_{0.5} \mathrm{Mn}_{0.5} \mathrm{TiO}_{3}$ is well described by a (short-range) three-dimensional Edwards-Anderson Ising spin glass. It would be of interest to further characterize other materials as well as effective models attempting to describe these using the FORC method. This is of paramount importance for materials for which model Hamiltonians are currently either unknown or under debate. For example, $\mathrm{Fe}_{0.85} \mathrm{Zn}_{0.15} \mathrm{~F}_{2}$ is a diluted antiferromagnet in a field ${ }^{35,36}$ which is expected to be well described by a random-field Ising model ${ }^{5,6}$ Currently experiments as well as simulations are being performed to characterize this material/model using the FORC method.

\section{Acknowledgments}

We would like to thank D. P. Belanger, F. Hassler, and G. T. Zimányi for helpful discussions. The simulations were performed on the hreidar cluster at ETH Zürich. This work has been supported in part by the Swiss National Science Foundation under Grant No. PP002114713, the EU-RTN project DYGLAGEMEM, and the Swedish Research Council.
${ }^{1}$ K. Binder and A. P. Young, Spin glasses: Experimental facts, theoretical concepts and open questions, Rev. Mod. Phys. 58, 801 (1986).

2 A. P. Young, ed., Spin Glasses and Random Fields (World Scientific, Singapore, 1998).

3 N. Kawashima and H. Rieger, Recent Progress in Spin Glasses (2003), (cond-mat/0312432).

4 J. Zhu, Micromagnetics of thin-film media, in Magnetic Recording Technology, edited by C. D. Mee and E. D. Daniel (Mc Graw Hill, New York, 1990).

5 J. P. Sethna, K. Dahmen, S. Kartha, J. A. Krumhansl,
B. W. Roberts, and J. D. Shore, Hysteresis and hierarchies: Dynamics of disorder-driven first-order phase transformations, Phys. Rev. Lett. 70, 3347 (1993).

6 I. F. Lyuksyutov, T. Nattermann, and V. Pokrovsky, Theory of the hysteresis loop in ferromagnets, Phys. Rev. B 59, 4260 (1999).

7 F. Pázmándi, G. Zaránd, and G. T. Zimányi, Selforganized criticality in the hysteresis of the sherringtonkirkpatrick model, Phys. Rev. Lett. 83, 1034 (1999).

8 H. G. Katzgraber, F. Pázmándi, C. R. Pike, K. Liu, R. T. Scalettar, K. L. Verosub, and G. T. Zimányi, Reversal-field 
memory in the hysteresis of spin glasses, Phys. Rev. Lett. 89, 257202 (2002).

${ }^{9}$ G. Bertotti, Hysteresis and Magnetism for Physicists, Materials Scientists, and Engineers (Academic Press, New York, 1998).

10 S. F. Edwards and P. W. Anderson, Theory of spin glasses, J. Phys. F: Met. Phys. 5, 965 (1975).

11 E. Della Torre, Magnetic Hysteresis (IEEE Press, New York, 1999).

12 C. R. Pike, A. P. Roberts, and K. L. Verosub, Characterizing interactions in fine magnetic particle systems using first order reversal curves, J. Appl. Phys. 85, 6660 (1999).

13 F. Preisach, Über die magnetische Nachwirkung, Z. Phys. 94, 277 (1935).

14 I. D. Mayergoyz, IEEE Trans. Magn. MAG-22 p. 603 (1986).

15 X. He, C. Alexander Jr., and M. R. Parker, IEEE Trans. Magn. MAG-28 p. 2683 (1992).

16 R. Proksh and B. Moskowitz, J. Appl. Phys. 75, 5894 (1994).

17 P. Hedja, E. Petrovsky, and T. Zelinka, IEEE Trans. Magn. MAG-30 p. 896 (1994).

18 X.-D. Che and H. N. Bertram, J. Magn. Magn. Mater. 116, 131 (1992).

19 M. El-Hilo, K. O'Grady, P. I. Mayo, and R. W. Chantrell, IEEE Trans. Magn. MAG-28 p. 3282 (1992).

20 For further details on the method and implementation see Ref. 12 .

21 H. G. Katzgraber, G. Friedman, and G. T. Zimányi, Fingerprinting hysteresis, Physica B 343, 10 (2004).

22 J. E. Davies, O. Hellwig, E. E. Fullerton, J. S. Jiang, S. D. Bader, and G. T. Zimanyi, Anisotropy Dependence of Irreversible Switching in $\mathrm{Fe} / \mathrm{SmCo}$ and $\mathrm{FeNi} / \mathrm{FePt}$ Exchange Spring Magnet Films, Appl. Phys. Lett. 86, 262503 (2005).

23 J. E. Davies, O. Hellwig, E. E. Fullerton, J. S. Jiang, S. D. Bader, G. T. Zimányi, and K. Liu, Anisotropy dependence of irreversible switching in $\mathrm{Fe} / \mathrm{SmCo}$ and $\mathrm{FeNi} / \mathrm{FePt}$ exchange spring magnet films, Appl. Phys. Lett. 86, 2503 (2005).

${ }^{24}$ I. Abou Hamad, D. T. Robb, and P. A. Rikvold, New Cyclic Voltammetry Method for Examining Phase Transitions: Simulated Results (2006), (cond-mat/0605601).
25 J. E. Davies, J. Wu, C. Leighton, and K. Liu, Magnetization reversal and nanoscopic magnetic-phase separation in $\mathrm{La}_{1-x} \mathrm{Sr}_{x} \mathrm{CoO}_{3}$, Phys. Rev. B 72, 134419 (2005).

26 C. Enachescu, R. Tanasa, A. Stancu, E. Codjovi, J. Linares, and F. Varret, FORC method applied to the thermal hysteresis of spin transition solids: first approach of static and kinetic properties, Physica B 343, 15 (2004).

27 A. Ito, E. Torikai, S. Morimoto, H. Aruga, M. Kikuchi, Y. Syono, and H. Takei, Behaviour of the transverse spin component in the short range ising spin-glass $\mathrm{Fe}_{0.5} \mathrm{Mn}_{0.5} \mathrm{TiO}_{3}$, J. Phys. Soc. Jpn. 59, 829 (1990).

28 A. Ito, C. Oyama, A. Fukaya, H. Kato, and S. Miura, Magnetization process of the mixed compound with exchange frustrations $\mathrm{Fe}_{0.65} \mathrm{Mn}_{0.35} \mathrm{TiO}_{3}$ and $\mathrm{Fe}_{0.5} \mathrm{Mn}_{0.5} \mathrm{TiO}_{3}$ - Anomalous dependence on the sweep rate of magnetic fields, J. Phys. Soc. Jpn. 66, 3636 (1997).

29 M. Östh, D. Hérisson, and P. Nordblad, Experimental first order reversal curves (FORC) on Ising and Heisenberg spin glasses, J. Magn. Magn. Mater. 310, e525 (2007).

30 H. G. Katzgraber, M. Körner, and A. P. Young, Universality in three-dimensional Ising spin glasses: A Monte Carlo study, Phys. Rev. B 73, 224432 (2006).

31 An updating scheme, where the spins are sorted by their degree of instability (size of the local field) and then flipped in order yields the same results. We use random updates since these are faster to implement.

32 N. Metropolis and S. Ulam, J. Am. Stat. Assoc. 44, 335 (1949).

33 R. H. Landau and M. J. Páez, Computational Physics (Wiley, New York, 1997).

34 See, for example, Ref. 30 for typical equilibration times.

35 F. Ye, L. Zhou, S. Larochelle, L. Lu, D. P. Belanger, M. Greven, and D. Lederman, Order Parameter Criticality of the $d=3$ Random-Field Ising Antiferromagnet $F_{0.85} Z n_{0.15} F_{2}$, Phys. Rev. Lett 89, 157202 (2002).

${ }^{36}$ F. Ye, L. Zhou, S. A. Meyer, L. J. Shelton, D. P. Belanger, L. Lu, S. Larochelle, and M. Greven, Quasistationary criticality of the order parameter of the three-dimensional random-field Ising antiferromagnet $\mathrm{Fe}_{0.85} Z_{0.15} \mathrm{~F}_{2}$ : A synchrotron x-ray scattering study, Phys. Rev. B 74, 144431 (2006). 